\title{
New Model-independent Method to Test the Curvature of Universe
}

\author{
H. $\mathrm{Yu}^{1}$ and F. Y. Wang ${ }^{1,2 *}$ \\ ${ }^{1}$ School of Astronomy and Space Science, Nanjing University, Nanjing 210093, China \\ ${ }^{2}$ Key Laboratory of Modern Astronomy and Astrophysics (Nanjing University), Ministry \\ of Education, Nanjing 210093, China \\ *fayinwang@nju.edu.cn
}

\begin{abstract}
We propose a new model-independent method to test the cosmic curvature by comparing the proper distance and transverse comoving distance. Using the measurements of Hubble parameter $H(z)$ and angular diameter distance $d_{A}$, the cosmic curvature parameter $\Omega_{K}$ is constrained to be $-0.09 \pm 0.19$, which is consistent with a flat universe. We also use Monte Carlo simulation to test the validity and efficiency, and find that our method can give a reliable and efficient constraint on cosmic curvature. Compared with other model-independent methods testing the cosmic curvature, our method can avoid some drawbacks and give a better constraint.
\end{abstract}

Subject headings: cosmological parameters - cosmology: observations

\section{Introduction}

The cosmic curvature is a fundamental parameter for cosmology. Whether the space of our Universe is open, flat, or closed is important for us to understand the evolution of our universe, and dark energy equation of state (Clarkson. Cortês. \& Bassett 2007; Zhao et al. 2007; Ichikawa et al. 2006; Weinberg et al. 2013). Due to the strong degeneracy between the curvature and the dark energy equation of state, it is difficult to study a non-flat $\omega \mathrm{CDM}$ model (Clarkson, Cortês, \& Bassett 2007). Besides, a significant detection of a nonzero curvature will affect the fundamental theory of cosmology because most observations support a flat $\Lambda \mathrm{CDM}$ model, including the latest Planck result which gives $\left|\Omega_{k}\right|<0.005$ (Planck Collaboration et al. 2015). However, most of these constraints are not in a direct geometric way. Therefore, determining the cosmic curvature with model-independent methods is very important. 
In order to constrain the cosmic curvature in a direct geometric way, some definitions of cosmological distance should be introduced. Several distance definitions, such as the proper distance $d_{P}$, luminosity distance $d_{L}$, angular diameter distance $d_{A}$ and transverse comoving distance $d_{M}$ are defined to investigate cosmology (Hogg 1999; Coles \& Lucchin 2002; Weinberg 2008; Weinberg et al. 2013). Under the assumption of Friedmann-LemaitreRobertson-Walker metric, the proper distance can be expressed as

$$
d_{P}(r)=a_{0} \int_{0}^{r} \frac{d r^{\prime}}{\sqrt{1-K r^{\prime 2}}}=a_{0} f(r)
$$

with $f(r)=\sin ^{-1} r, r$, or $\sinh ^{-1} r$ for curvature $K=+1,0$, or $-1, a_{0}$ is the present scale factor, and $r$ is the comoving coordinate of the source. With the definition of Hubble parameter $H(z)=\dot{a} / a$, it can also be expressed as

$$
d_{P}(z)=\frac{c}{H_{0}} \int_{0}^{z} \frac{d z^{\prime}}{E\left(z^{\prime}\right)}
$$

where $z$ is the redshift, $H_{0}$ is the Hubble constant, $c$ is the speed of light and $E(z)=H(z) / H_{0}$. Similarly, the transverse comoving distance can be expressed as

$$
d_{M}(z)=a_{0} r(z)=\frac{c}{H_{0} \sqrt{-\Omega_{K}}} \sin \left[\sqrt{-\Omega_{K}} \int_{0}^{z} \frac{d z^{\prime}}{E\left(z^{\prime}\right)}\right],
$$

where $\Omega_{K}$ is the energy density of cosmic curvature $\left(-i \sin (i x)=\sinh (x)\right.$ if $\left.\Omega_{K}>0\right)$. With the definition of $d_{M}, d_{L}$ and $d_{A}$ can be derived through $d_{L}=d_{M}(1+z)$ and $d_{A}=d_{M} /(1+z)$, respectively.

Numerous works have been done to determine the curvature parameter $\Omega_{K}$ using different methods, some of which are model-independent. Bernstein (2006) proposed a modelindependent method using the weak lensing and baryon acoustic oscillation (BAO) data to constrain $\Omega_{K}$ based on the distance sum rule, which was used to test the FLRW metric in Räsänen et al. (2015) (hereafter, called DSR method). The basic principle of DSR method is that the relation between $d\left(z_{s}\right)$ and $d\left(z_{l}\right)+d\left(z_{l}, z_{s}\right)$ depends on the cosmic geometry (see Fig. 1 of Bernstein $(2006))$. The value of $d\left(z_{l}, z_{s}\right)$ can be calculated from gravitational lensing. However, the large uncertainty in gravitational lens system restricts its efficiency on constraining the curvature (Räsänen et al. 2015). Another important model-independent method was proposed in Clarkson, Cortês, \& Bassett (2007), by comparing the Hubble parameter $H(z)$ and the derivative function of transverse comoving distance $d_{M}$ gained from $d_{A}$, which has been used in many works (Clarkson et al. 2008; Yahya et al. 2014; Li et al. 2014; Cai et al. 2016) (hereafter, C07 method). The basis of this method is that one can determine the curvature by combining measurements of the Hubble parameter $H(z)$ and the 
transverse comoving distance $d_{M}(z)$

$$
\Omega_{K}=\frac{\left[H(z) d_{M}^{\prime}(z)\right]^{2}-c^{2}}{\left[H_{0} d_{M}(z)\right]^{2}},
$$

where' means the derivative with respect to redshift $z$. However, in this method, one needs to determine the derivative function of transverse comoving distance $d_{M}$ from a fitting function, which will introduce a large uncertainty.

Therefore, in order to avoid the drawbacks of the two methods, we propose a new direct geometric method to test the cosmic curvature. This method is based on the comparison between proper distance $d_{P}$ obtained from Hubble parameter measurement, and transverse comoving distance $d_{M}$ obtained from angular diameter distance $d_{A}$ measurement. The structure of this paper is organized as follows. In section 2 , we introduce our new modelindependent method to test the cosmic curvature using $d_{P}$ and $d_{M}$. In section 3 , we give our constraint on $\Omega_{K}$ using the Hubble parameter and angular diameter distance measurements. In section 4, we test the validity and efficiency of our method with Monte Carlo simulation. In section 5, we discuss its advantages compared with other methods. Finally a summary will be given in section 6 .

\section{Method to test $\Omega_{K}$}

Comparing the definitions of proper distance $d_{P}$ and transverse comoving distance $d_{M}$, one can find that the difference between them only caused by the curvature of universe. This gives the basis to test the cosmic curvature using the comparison of $d_{P}$ and $d_{M}$. From equations (2) and (3), $\Omega_{K}$ can be derived from

$$
\frac{H_{0} d_{M}}{c} \sqrt{-\Omega_{K}}=\sin \left(\frac{H_{0} d_{P}}{c} \sqrt{-\Omega_{K}}\right)
$$

Equation (5) gives the direct relation among $\Omega_{K}, d_{P}$ and $d_{M}$. Once the $d_{P}$ and $d_{M}$ are determined, $\Omega_{K}$ can be calculated through this equation. If the value of redshift and $\Omega_{K}$ are not large, $H_{0} d_{P} \sqrt{-\Omega_{K}} / c$ is less than one. From the Taylor expansion, the equation (5) can be approximated as

$$
\Omega_{K}=\frac{6 c^{2}}{H_{0}^{2}} \frac{d_{M}-d_{P}}{d_{P}^{3}},
$$

from which $\Omega_{K}$ can be determined directly.

Figure 1 shows the key principle of our method in the $\Omega_{K}<0$ case. In this figure, the arc OS is the proper distance between source $S$ and observer $O$, while the transverse 
comoving distance between them is $d_{M}=a_{0} \sin \left(\frac{d_{P}}{a_{0}}\right)$. In this case, it is obvious that the $d_{M}$ of an object has an up limit $a_{0}$ and it is less than $d_{P}$. In contrast, in an open universe $\left(\Omega_{K}>0\right)$, we have $d_{M}>d_{P} . d_{M}=d_{P}$ only happens in a flat universe. Therefore, the cosmic curvature can be derived by comparing $d_{M}$ and $d_{P}$. Undering the assumption of $\sqrt{\left|\Omega_{K}\right|} I \ll 1$, we can obtain

$$
\delta \equiv \frac{d_{P}-d_{M}}{d_{P}}=-\frac{\Omega_{K} I^{2}}{6}
$$

with $I=\int_{0}^{z} \frac{d z^{\prime}}{E(z)}$. The $\delta$ means the relative difference between $d_{P}$ and $d_{M}$. The value of $|\delta|$ gives the requirement on the accuracy of measurement, which means the $\Omega_{K}$ cannot be constrained if the observed uncertainty is much larger than $|\delta|$. Besides, if the total relative error of the measurement sample of $d_{M}$ and $d_{P}$ is $\sigma$, one can expect that the tightest constrain on $\Omega_{K}$ will have an error about $\sigma_{\Omega_{K}} \sim 6 \sigma / I^{2}$. In other words, equation (7) gives the constraint limit of this method.

To obtain the transverse distance $d_{M}$, we choose the angular diameter distance $d_{A}$ measurement based on BAO in several previous works (Blake et al. 2012; Xu et al. 2013; Samushia et al. 2014; Delubac et al. 2015). These data and their references are listed in Table 1. The detailed information about these data can be found in their references. The $d_{M}$ can be easily derived with the direct relation between them $d_{M}=d_{A}(1+z)$. The next important issue is how to measure $d_{P}$. From equation (2), the proper distance $d_{P}$ only depends on the $H(z)$ function. Therefore, in order to derive the proper distance $d_{P}$, one can construct the $H(z)$ function from Hubble parameter measurements. Then $d_{P}$ can be derived from equation (2). There are tens of Hubble parameter measurements derived from differential ages of galaxies and the radial BAO in the previous literature, which are listed in Table 2. In order to make our method model-independent, Gaussian Process (GP) method is used to reconstruct the $H(z)$ function. GP method is a powerful tool to reconstruct a function from data directly without any assumption of the function form and is used widely in astronomy (Holsclaw et al. 2010; Shafieloo \& Clarkson 2010; Shafieloo et al. 2012; Seikel et al. 2012a; Bilicki \& Seikel 2012). Therefore, with GP method, we don't need any prior cosmological model. There is a good python package for GP method called Gapp developed by Seikel et al. (2012a) which was used in many works (Seikel et al. 2012b; Bilicki \& Seikel 2012; Cai et al. 2016). It can reconstruct the function as long as observed data was input. More detailed information about GP method and Gapp can be found in Seikel et al. (2012a).

The main route of our method is that: I) deriving the transverse comoving distance $d_{M}$ from angular diameter distance $d_{A}$ measurements; II) reconstructing $H(z)$ function from Hubble parameter measurements using GP method; III) using equation (2) to calculate the proper distance at a certain redshift; IV) using equation (15) or (6) to determine $\Omega_{K}$ at a certain redshift. Several $\Omega_{K}$ can be determined at different redshifts since there are several 
$d_{A}$ measurements. One can choose the average of them as the final result through

$$
\Omega_{K}=\frac{\sum_{i} \Omega_{K, i} / \sigma_{\Omega_{K, i}}^{2}}{\sum_{i} 1 / \sigma_{\Omega_{K, i}}^{2}}
$$

where $\Omega_{K, i}$ and $\sigma_{\Omega_{K, i}}$ are determined $\Omega_{K}$ and its uncertainty at a certain redshift. The total uncertainty can be obtained from

$$
\sigma_{\Omega_{K}}^{2}=\frac{1}{\sum_{i} 1 / \sigma_{\Omega_{K, i}}^{2}} .
$$

\section{Results}

We collect 31 Hubble parameter measurements from previous literature and list them in table 2. These Hubble parameters at different redshifts are derived using differential ages of galaxies and the radial BAO method. Using these observed data and GP method, the $H(z)$ function can be reconstructed, which is shown as the blue curve in Figure 2. Hereafter, this $H(z)$ function is called GP- $H(z)$. For comparison, we also fit the observed data based on $\Lambda \mathrm{CDM}$ model which is shown as the green curve in Figure 2. Hereafter, this $H(z)$ function is called Fit- $H(z)$. We can find that the Fit- $H(z)$ is well covered by the GP- $H(z)$ function and its $1 \sigma$ confidence region. With the reconstructed GP- $H(z)$ function, one can use equation (2) to derive the proper distance at a certain redshift. The derived $d_{P}(z)$ functions from GP- $H(z)$ and Fit- $H(z)$ are shown in Figure 3. Hereafter, they are called GP- $d_{P}(z)$ and Fit$d_{P}(z)$ respectively. Comparing the GP- $H(z)$ and Fit- $H(z)$, one can find that the derivation between them becomes smaller than that between GP-H(z) and Fit- $H(z)$.

Using equation (5), one can derive the $\Omega_{K}$ from the $d_{P}$ and $d_{M}$ at the same redshift. Figure 4 shows the result of derived $\Omega_{K}$ at different redshifts. The average $\Omega_{K}$ and its error bar are derived from equations (8) and (9). The result is listed in Table 3. The average $\Omega_{K}$ constrained by these six $d_{A}$ data is $\Omega_{K}=-0.09 \pm 0.19$. There is no significant deviation from a flat universe. From bottom panel of Figure 2, it is obvious that the high-redshift measurement gives tighter constraint on $\Omega_{K}$ than the low-redshift measurement. The reason is that the $I$ term in equation (7) is larger at high redshift, which will decrease the error of $\Omega_{K}$ through $\sigma_{\Omega_{K}} \sim 6 \sigma / I^{2}$. 


\section{Simulation}

In order to test the validity and efficiency of our method, we perform Monte Carlo simulation. The route of simulation is as follows: I) creating mock $H(z)-z$ and $d_{M}-z$ data sets based on a prior cosmological model; II) reconstructing the GP- $H(z)$ function from GP method; III) using GP- $H(z)$ function to derive the $\operatorname{GP}-d_{P}(z)$ function through equation (2); IV) using equations (5) and (8) to constrain $\Omega_{K}$ and its average value; V) simulating $10^{4}$ times for each prior cosmological model and give the distribution of determined average $\Omega_{K}$.

For simualtion, we choose $\Lambda$ CDM model as the prior cosmological model. The model parameters are chosen as $H_{0}=70 \mathrm{~km} / \mathrm{s} / \mathrm{Mpc}, \Omega_{M}=0.3$ and $\Omega_{\Lambda}=1-\Omega_{M}-\Omega_{K}$ where $\Omega_{K}=-0.1,0$ and 0.1 for different cases. In each simulation, there are 20 mock $H-z$ and $d_{M}-z$ data sets respectively. The redshifts of these mock data are chosen equally in $\log (1+z)$ space in redshift range $0.1 \leq z \leq 5.0$. The relative uncertainty of these mock data is $1 \%$ which will be realized in future observation (Weinberg et al. 2013).

Figure 5 gives an example of the simulations in the $\Omega_{K}=0$ case. The three panels show the mock Hubble parameter data with GP-H(z) function, mock $d_{M}$ data with GP$d_{P}(z)$ function and the final determined $\Omega_{K}$. From this figure, we can see that GP method can reconstruct $H(z)$ function well. In this case, the final derived average $\Omega_{K}$ is $\Omega_{K}=$ $0.0001 \pm 0.0092$. Figure 6 shows the posterior distributions of $\Omega_{K}$ for three $\Omega_{K}$ cases. From this figure, one can find that our method can give a reliable and tight constraint on the prior $\Omega_{K}$. The uncertainty is $\sigma_{\Omega_{K}} \approx 0.011$. This result means that if there are $20 d_{A}$ and $H(z)$ measurements with $1 \%$ uncertainty, our method can give a constraint on $\Omega_{K}$ at $1 \%$ level. In future, there will be more accurate measurements of $d_{A}$ and $H(z)$, tighter constraint on $\Omega_{K}$ can be expected.

\section{Compared with other methods}

In this section, we compare our method with other model-independent methods. Just as introduced in the first section, there are two model-independent methods to constrain the curvature of universe proposed in previous literature (Bernstein 2006; Clarkson. Cortês, \& Bassett 2007), which have been used in many works (Clarkson et al. 2008; Yahya et al. 2014; Li et al. 2014; Räsänen et al. 2015; Cai et al. 2016). The first one is DSR method based on the distance sum rule proposed by Bernstein (2006) and the other is C07 method based on the equation (4) (Clarkson, Cortês, \& Bassett 2007).

The basic principle of DSR method is the distance sum rule which means that if there are two sources $S_{1}$ and $S_{2}$ at redshift $z_{1}$ and $z_{2}$ (assuming $\left.z_{1}<z_{2}\right)$, it has $d\left(z_{2}\right)=d\left(z_{1}\right)+d\left(z_{1}, z_{2}\right)$ 
if our universe is flat. Otherwise, $d\left(z_{2}\right)>d\left(z_{1}\right)+d\left(z_{1}, z_{2}\right)$ or $d\left(z_{2}\right)<d\left(z_{1}\right)+d\left(z_{1}, z_{2}\right)$ for $\Omega_{K}>0$ or $\Omega_{K}<0$ respectively. The distance between $S_{1}$ and $S_{2}$ can be determined by gravitational lens (Bernstein 2006; Räsänen et al. 2015). Compared the Figure 1 of Bernstein (2006) with our Figure 1, it can be found that the difference between $d_{P}$ and $d_{M}$ is larger than that between $d\left(z_{1}\right)+d\left(z_{1}, z_{2}\right)$ and $d\left(z_{2}\right)$, which means our method is more sensitive on the cosmic curvature. An accurate calculation gives the relative difference between $d\left(z_{1}\right)+d\left(z_{1}, z_{2}\right)$ and $d\left(z_{2}\right)$ is

$$
\left|\delta_{D S R}\right| \leq\left|\frac{\Omega_{K} I^{2}}{8}\right|,
$$

where $=$ is only valid when the source $S_{1}$ locates at the middle of $S_{2}$ and observer. Compared with equation (7), one can find that DSR method needs a higher accuracy of measurement than our method. Besides, the systematic uncertainty of gravitation lens system parameter $f^{2}$ is about $20 \%$ (Kochanek et al. 2000), where $f$ is a phenomenological coefficient that parameterizes uncertainty due to difference between the velocity dispersion of the observed stars and the underlying dark matter, and other systematic effects in strong lensing. This systematic uncertainty is hardly to remove, which restricts its efficiency on constraining the cosmic curvature.

For the $\mathrm{C} 07$ method, it is based on equation (4), which needs the first derivative function of $d_{M}(z)$ fitted from observational data. As is known to all that calculating the derivative

function from a fitted function will increase the uncertainty significantly especially when the function form is unknown and the data is not enough. In order to check the efficiency of C07 method, we also use Monte Carlo simulation to test it, and the simulation is same as introduced in section 4. Figure 7 gives an example of the simulations in the $\Omega_{K}=0$ case, which is similar with Figure 5, Instead of $H(z)$ and $d_{P}(z)$ functions, we show the $d_{M}^{\prime}(z)$ function in the top panel of Figure 7. Because the $H(z)$ and $d_{P}(z)$ functions are similar with those in Figure 5. From Figure 7, it is obvious that the $d_{M}^{\prime}(z)$ function derived from GP method has a large derivation with the theoretical one, and the determined $\Omega_{K}$ are not as well as those in bottom panel of Figure 5 . Figure 8 shows the posterior distributions of $\Omega_{K}$ determined with $\mathrm{C} 07$ method for three $\Omega_{K}$ cases. From this figure, one can find that C07 method gives a large uncertainty on the determined $\Omega_{K}$.

\section{Summary}

We have proposed a new model-independent method to test the cosmic curvature in this paper. The main principle of our method is to compare the proper distance $d_{P}$ and transverse comoving distance $d_{M}$ at same redshift (Figure 1 gives an illustration). Using 
equation (5), one can derive the $\Omega_{K}$ if $d_{P}$ and $d_{M}$ are obtained. With the measurements of Hubble parameter, we use GP method to reconstruct the $H(z)$ function and use equation (2) to derive the $d_{P}(z)$ function. Using the measurements of angular diameter distance, transverse comoving distance can be calculated easily through $d_{M}=d_{A}(1+z)$. We used the $H(z)$ and $d_{A}$ measurements collected from previous literature. The reconstructed $H(z)$ function and the derived $d_{P}(z)$ function are shown in Figures 2 and 3 . In order to compare with $\Lambda$ CDM model, the best-fitted $H(z)$ and $d_{P}(z)$ function are also shown in these figures. The comparison shows that the GP method can give a reliable reconstructed function from observed data. Figure 4 shows the derived $\Omega_{K}$ at several different redshifts, which are also listed in Table 3. Using equations (8) and (9), the average $\Omega_{K}$ can be obtained, which is $\Omega_{K}=-0.09 \pm 0.19$. This result shows that $\Omega_{K}$ has no significant derivation from non-zero value.

To check the validity and efficiency of our method, we use Monte Carlo simulation to test it. For $\Lambda$ CDM model with three different $\Omega_{K},-0.1,0$ and 0.1 , we simulate $10^{4}$ times for each case. Figure 5 gives an example of the simulations for $\Omega_{K}=0$ case and Figure 6 gives the posterior distributions of $\Omega_{K}$ determined with our method for the three $\Omega_{K}$ cases. These two figures show that our method can give a reliable and efficient constraint on $\Omega_{K}$. We also compared our method with the DSR and C07 method. We find that DSR method needs a higher accuracy of measurement than our method. More importantly, the systematic uncertainty of gravitational lens system parameter $f^{2}$ restricts significantly its efficiency on constraining the curvature. For the C07 method, we also test it with simulations. The result is shown in Figures 7 and 8, From Figure 7, we find that the first derivative function of $d_{M}(z)$ derived from GP method has a large derivation with the theoretical one. So the determined $\Omega_{K}$ not reliable. Figure 8 shows the posterior distributions of $\Omega_{K}$ determined by C07 method for the three $\Omega_{K}$ cases. Meanwhile, the C07 method will give a large uncertainty on the determined $\Omega_{K}$ with observed data at same accuracy level.

Future observations will improve the constraint on the cosmic curvature. The Extended Baryon Oscillation Spectroscopic Survey (eBOSS) will complie 250,000 new, spectroscopically confirmed luminous red galaxies, which yield measurements of $d_{A}$ with $1.2 \%$ precision and measurements of $H(z)$ with $2.1 \%$ precision (Dawson et al. 2016). HETDEX will perform a survey of 800,000 Ly $\alpha$ emission-line galaxies at $1.8<z<3.7$ (Hill et al. 2006). The precision on $d_{A}$ and $H(z)$ is of order $2 \%$ using BAO. The BAO analysis from Wide Field Infrared Survey Telescope (WFIRST) will yield about $1.0 \%$ measurements of the angular diameter distance $d_{A}$ and Hubble parameter $H(z)$ by 17 million galaxies redshift survey in the redshift range $1.3<z<2.7$ (Green et al. 2012). Meanwhile, the Euclid satellite with survey area of approximately 14,000 $\mathrm{deg}^{2}$ and redshift range $0.7<z<2.0$ will measure Hubble parameter $H(z)$ with $1.5 \%$ precision (Laureijs et al. 2011). Weinberg et al. (2013) had predicted the 
accuracy of future measurement of Hubble parameter and angular diameter distance through the full sky BAO survey and gave a encourage forecast that the relative error on $H(z)$ and $d_{A}$ would be less than $1 \%$ at redshift $z>0.5$. Therefore, with our method, the curvature parameter $\Omega_{K}$ can be constrained at a very high accuracy level in a model-independent way.

\section{Acknowledgements}

We thank the anonymous referee for useful comments and suggestions. This work is supported by the National Basic Research Program of China (973 Program, grant No. 2014CB845800) and the National Natural Science Foundation of China (grants 11422325 and 11373022), the Excellent Youth Foundation of Jiangsu Province (BK20140016).

\section{REFERENCES}

Bernstein, G. 2006, ApJ, 637, 598

Bilicki, M., \& Seikel, M. 2012, MNRAS, 425, 1664

Blake, C., Brough, S., Colless, M., et al. 2012, MNRAS, 425, 405

Cai, R.-G., Guo, Z.-K., \& Yang, T. 2016, Phys. Rev. D, 93, 043517

Clarkson C., Cortês M., Bassett B., 2007, JCAP, 8, 011

Clarkson, C., Bassett, B., \& Lu, T. H.-C. 2008, Physical Review Letters, 101, 011301

Coles, P., \& Lucchin, F. 2002, Cosmology: The Origin and Evolution of Cosmic Structure, Second Edition, by Peter Coles, Francesco Lucchin, pp. 512. ISBN 0-471-489093. Wiley-VCH , p13, July 2002

Dawson, K. S., Kneib, J.-P., Percival, W. J., et al. 2016, AJ, 151, 44

Delubac, T., Bautista, J. E., Busca, N. G., et al. 2015, A\&A, 574, A59

Gaztañaga, E., Cabré, A., \& Hui, L. 2009, MNRAS, 399, 1663

Green, J., Schechter, P., Baltay, C., et al. 2012, arXiv:1208.4012

Hill, G. J., MacQueen, P. J., Palunas, P., et al. 2006, New A Rev., 50, 378

Hogg, D. W. 1999, arXiv:astro-ph/9905116 
Holsclaw, T., Alam, U., Sansó, B., et al. 2010, Physical Review Letters, 105, 241302

Ichikawa K., Kawasaki M., Sekiguchi T., Takahashi T., 2006, JCAP, 12, 005

Ichikawa K., Takahashi T., 2006, PhRvD, 73, 083526

Jimenez, R., Verde, L., Treu, T., \& Stern, D. 2003, ApJ, 593, 622

Kochanek, C. S., Falco, E. E., Impey, C. D., et al. 2000, ApJ, 543, 131

Laureijs, R., Amiaux, J., Arduini, S., et al. 2011, arXiv:1110.3193

Li, Y.-L., Li, S.-Y., Zhang, T.-J., \& Li, T.-P. 2014, ApJ, 789, L15

Moresco, M., Verde, L., Pozzetti, L., Jimenez, R., \& Cimatti, A. 2012, JCAP, 7, 053

Moresco, M. 2015, MNRAS, 450, L16

Planck Collaboration, Ade, P. A. R., Aghanim, N., et al. 2015, arXiv:1502.01589

Räsänen, S., Bolejko, K., \& Finoguenov, A. 2015, PRL, 115, 101301

Samushia, L., Reid, B. A., White, M., et al. 2014, MNRAS, 439, 3504

Seikel, M., Clarkson, C., \& Smith, M. 2012a, J. Cosmology Astropart. Phys., 6, 036

Seikel, M., Yahya, S., Maartens, R., \& Clarkson, C. 2012b, Phys. Rev. D, 86, 083001

Shafieloo, A., \& Clarkson, C. 2010, Phys. Rev. D, 81, 083537

Shafieloo, A., Kim, A. G., \& Linder, E. V. 2012, Phys. Rev. D, 85, 123530

Simon, J., Verde, L., \& Jimenez, R. 2005, Phys. Rev. D, 71, 123001

Stern, D., Jimenez, R., Verde, L., Kamionkowski, M., \& Stanford, S. A. 2010, J. Cosmology Astropart. Phys., 2, 008

Xu, X., Cuesta, A. J., Padmanabhan, N., Eisenstein, D. J., \& McBride, C. K. 2013, MNRAS, 431,2834

Weinberg, S. 2008, Cosmology, by Steven Weinberg. ISBN 978-0-19-852682-7. Published by Oxford University Press, Oxford, UK, p24, 2008.

Weinberg, D. H., Mortonson, M. J., Eisenstein, D. J., et al. 2013, Phys. Rep., 530, 87 
Yahya, S., Seikel, M., Clarkson, C., Maartens, R., \& Smith, M. 2014, Phys. Rev. D, 89, 023503

Zhang, C., Zhang, H., Yuan, S., et al. 2014, Research in Astronomy and Astrophysics, 14, 1221-1233

Zhao G.-B., Xia J.-Q., Li H., Tao C., Virey J.-M., Zhu Z.-H., Zhang X., 2007, PhLB, 648, 8 


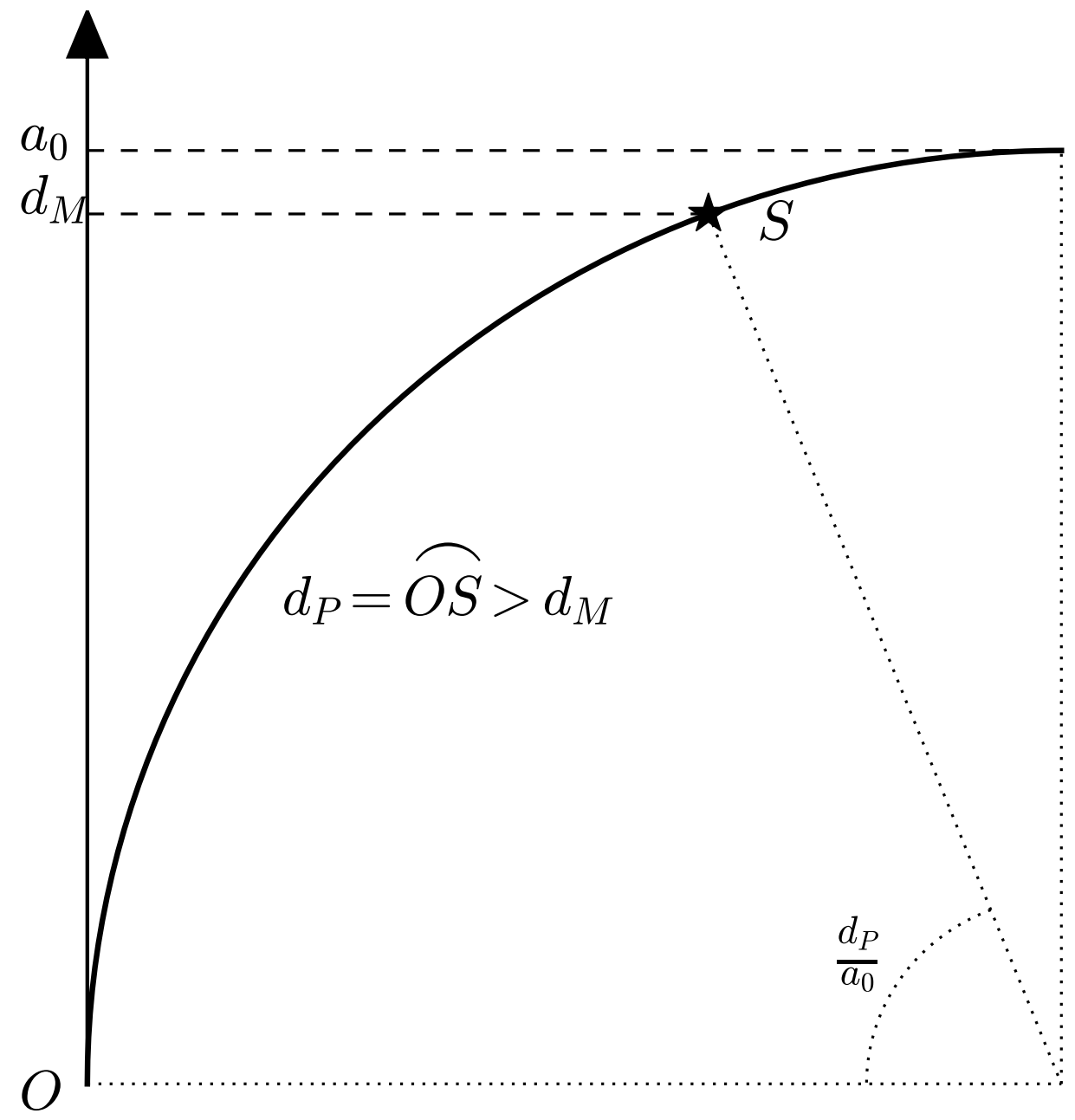

Fig. 1.- Illustration of the proper distance $d_{P}$ and transverse comoving distance $d_{M}$ in a closed universe. It is obvious that $d_{M}<d_{P}$. 


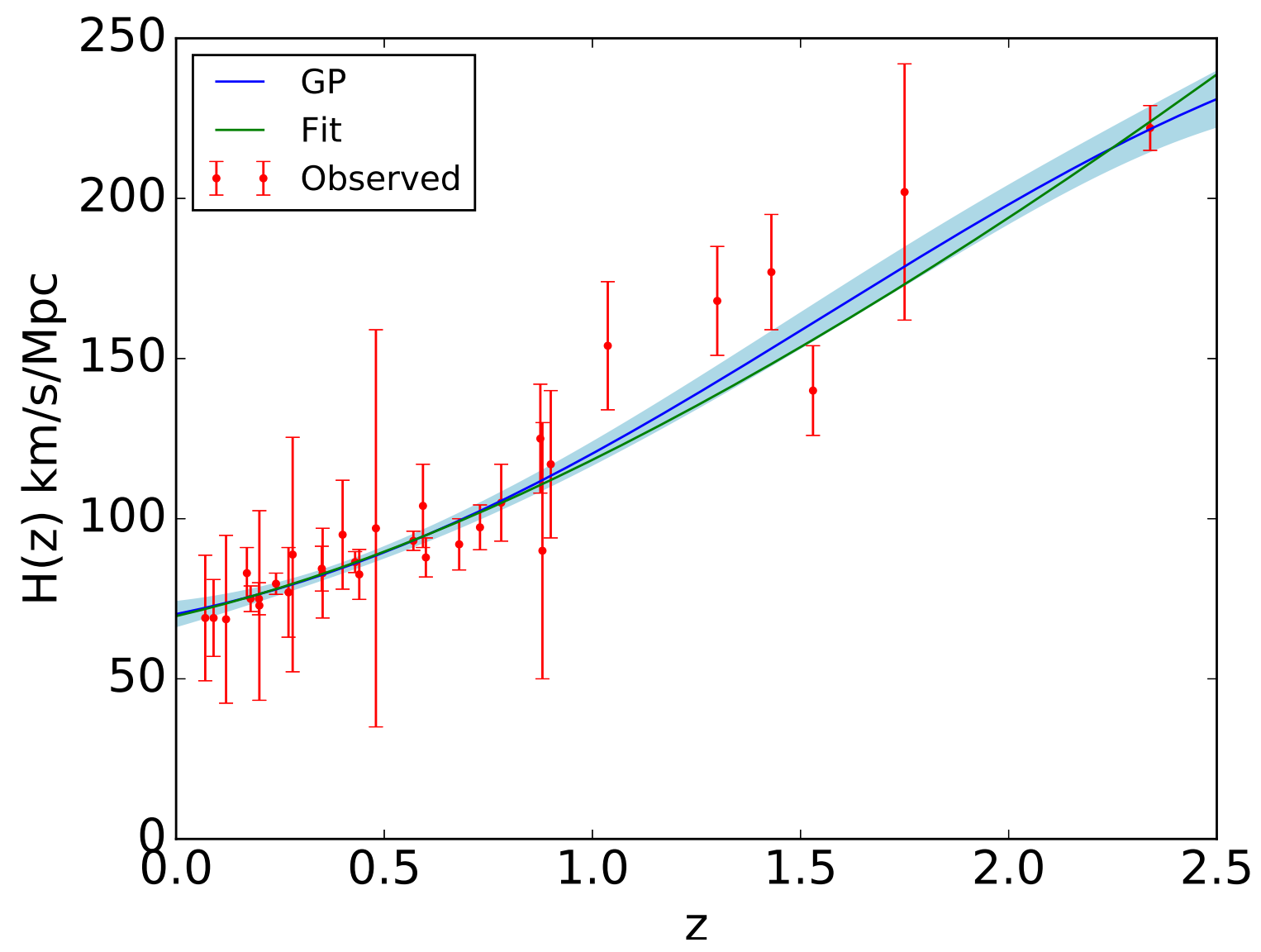

Fig. 2.- The blue curve and area show the $H(z)$ function and its $1 \sigma$ confidence region reconstructed from GP method. The green curve shows the $H(z)$ function fitted with $\Lambda \mathrm{CDM}$ model. The red points and the error bars show the observed Hubble parameters and their $1 \sigma$ errors. 


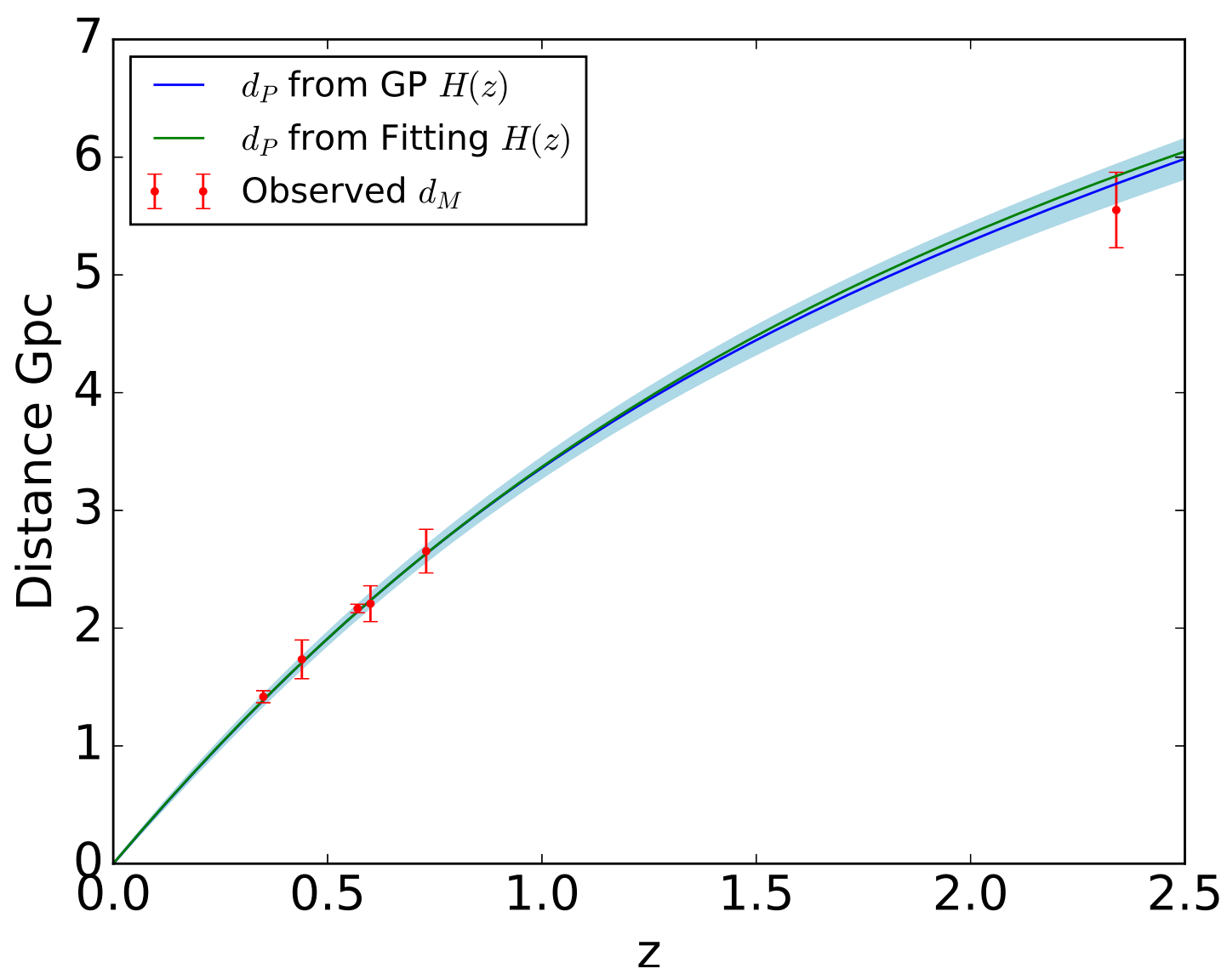

Fig. 3.- The blue curve and area show the $d_{P}(z)$ function and its $1 \sigma$ confidence region derived from the GP- $H(z)$ function. The green curve shows the $d_{P}(z)$ function derived from the fitted $H(z)$ function. The red points and the error bars show the observed $d_{M}$ and their $1 \sigma$ errors. 


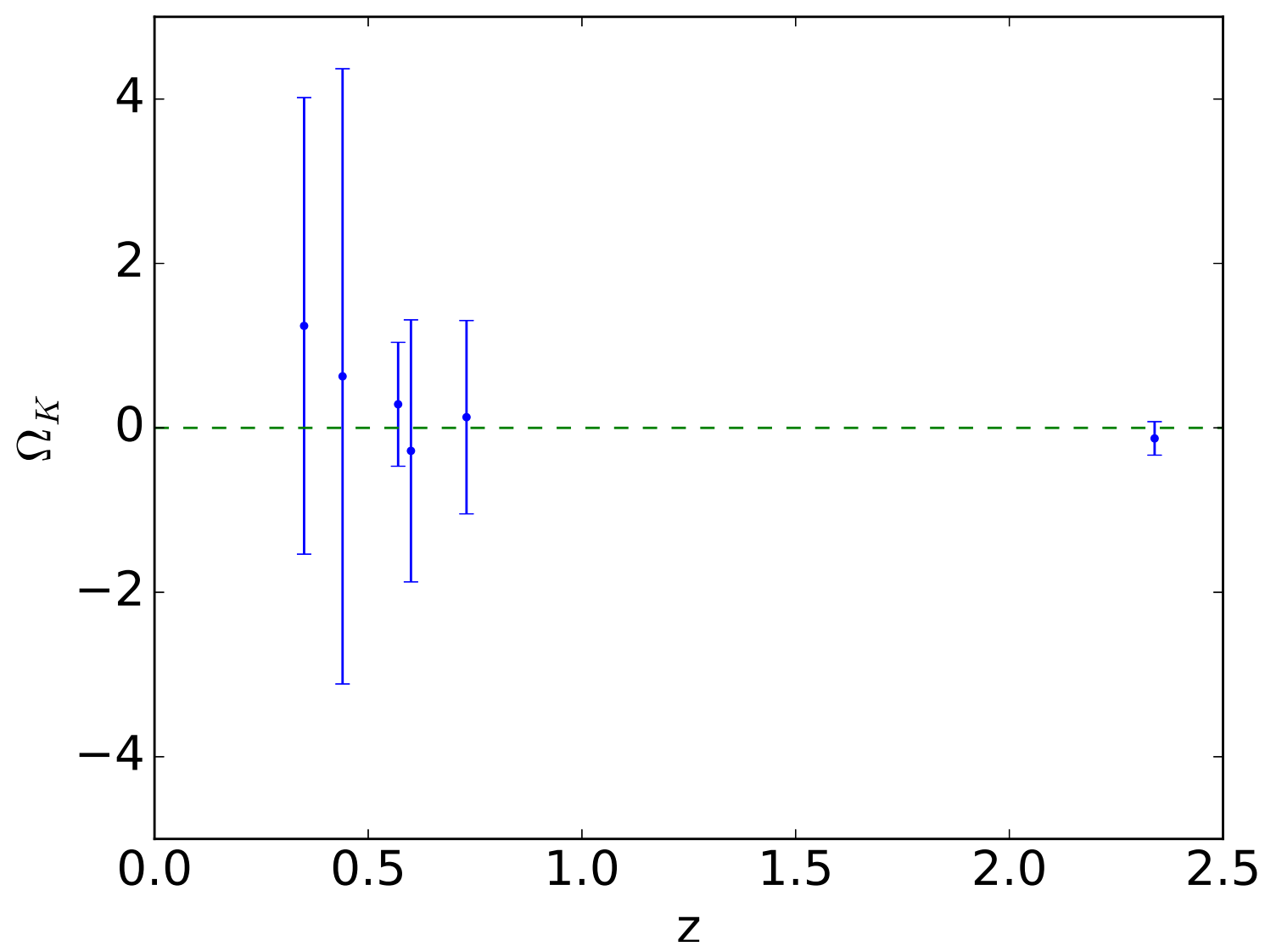

Fig. 4.- The $\Omega_{K}$ determined by comparing the $\operatorname{GP}-d_{P}(z)$ function and observed $d_{M}$. 

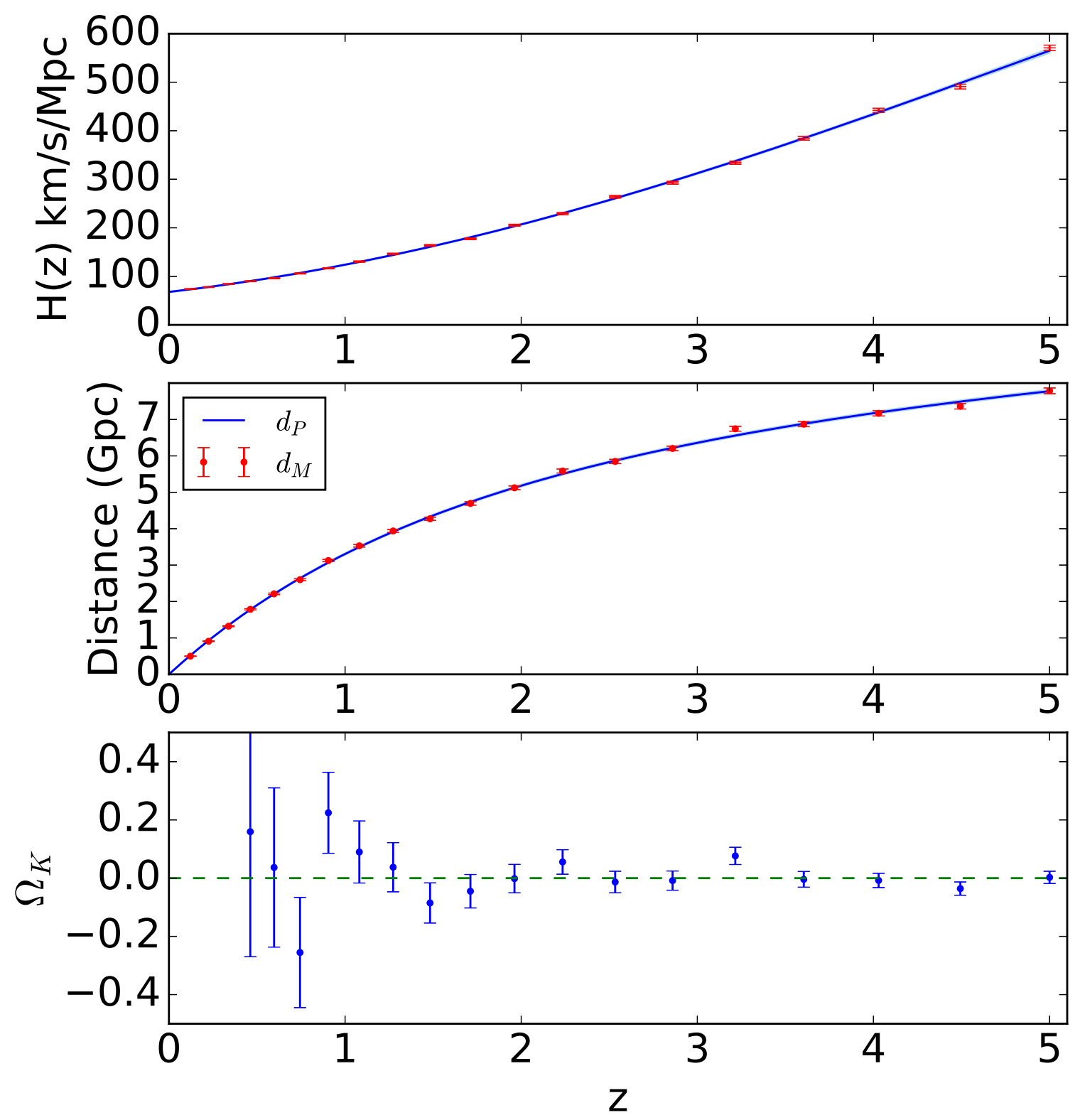

Fig. 5.- An example of the simulation for $\Omega_{K}=0$ case. Top panel shows the mock Hubble parameter data and the GP-H(z) function. Middle panel shows the mock $d_{M}$ data and the GP- $d_{P}(z)$ function. The bottom panel shows the final $\Omega_{K}$ determined from these mock data. 


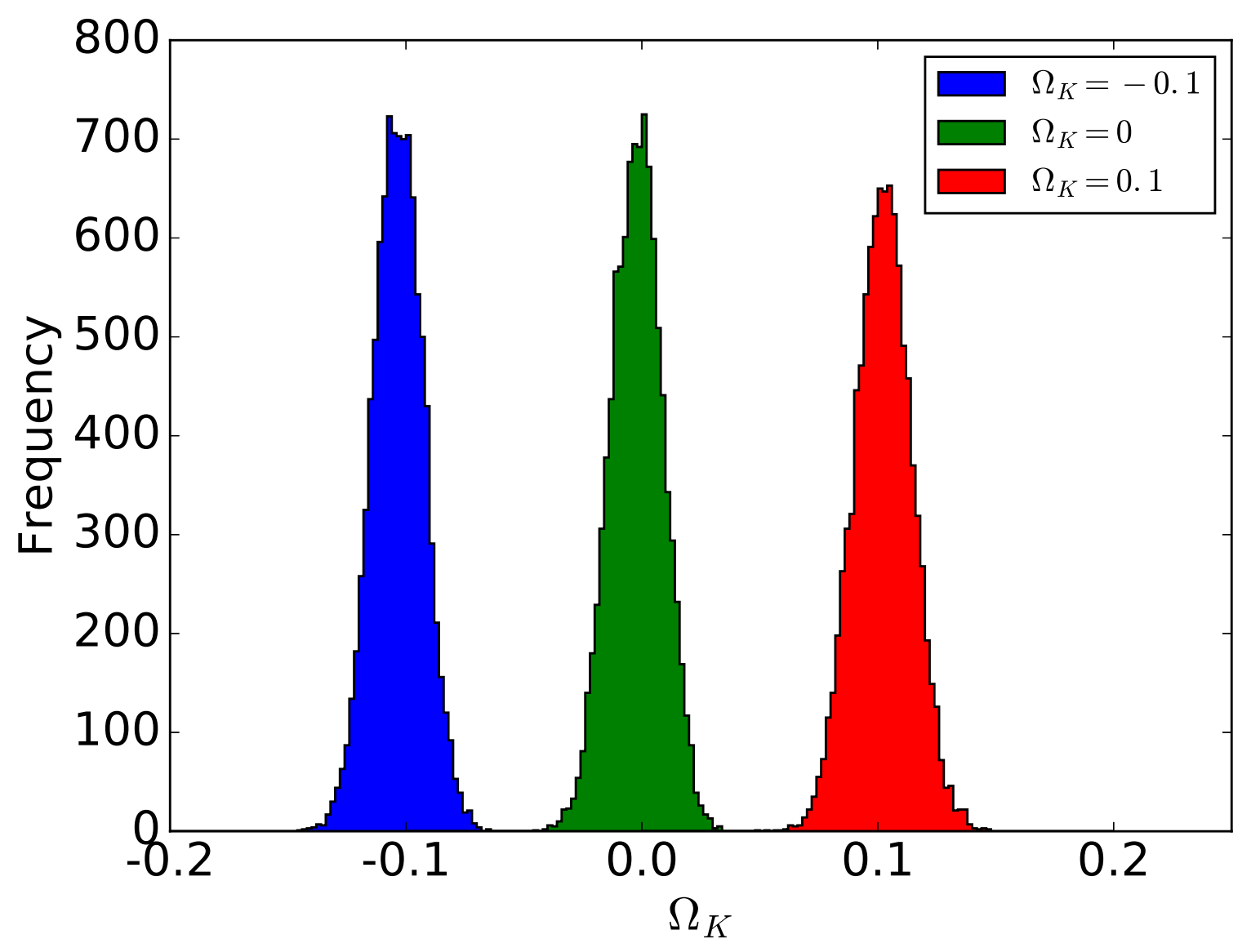

Fig. 6. - The distributions of $\Omega_{K}$ determined from simulated mock data based on background $\Lambda \mathrm{CDM}$ model with different prior curvatures using our method. 


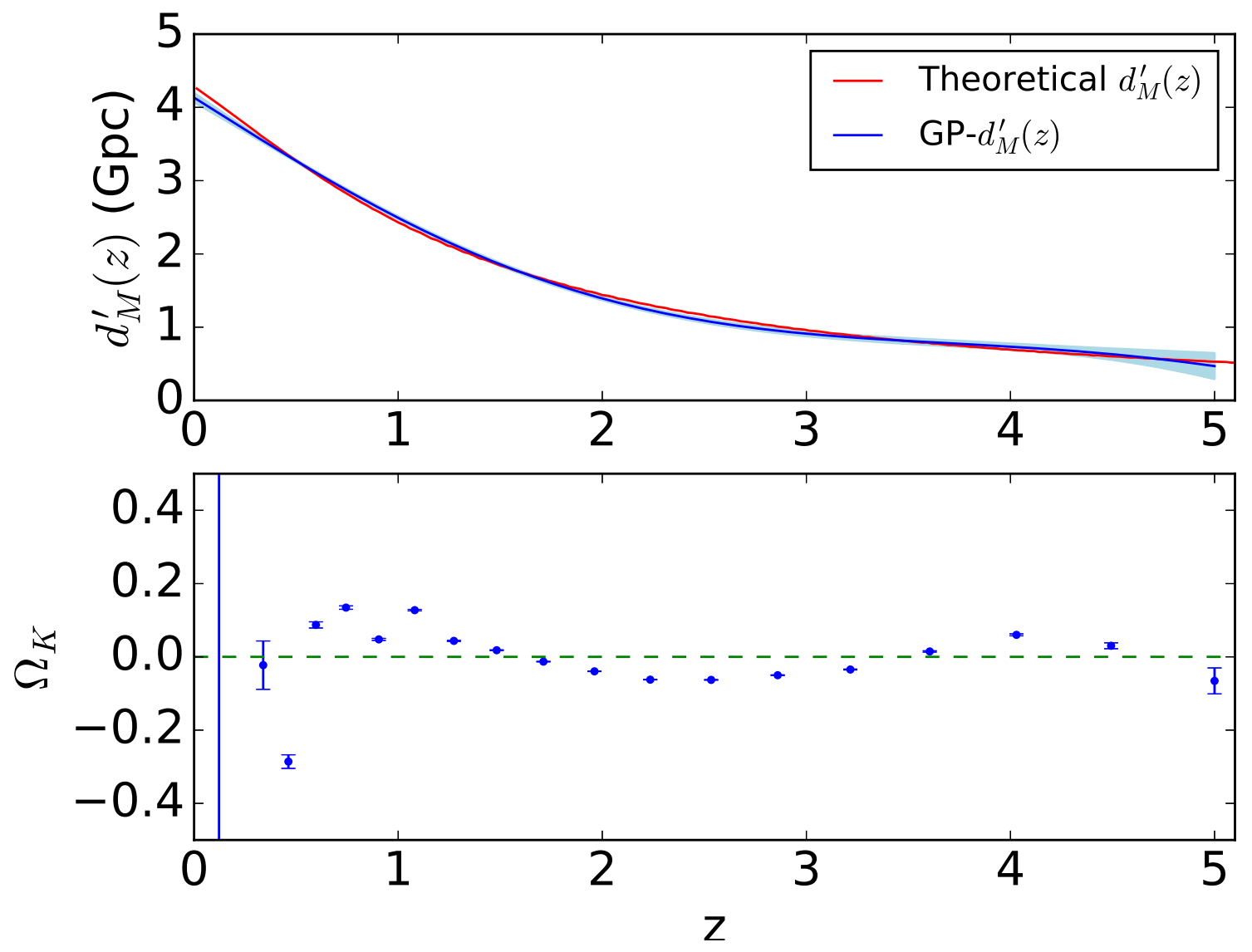

Fig. 7.- Same as Fig. 5 but for C07 method. 


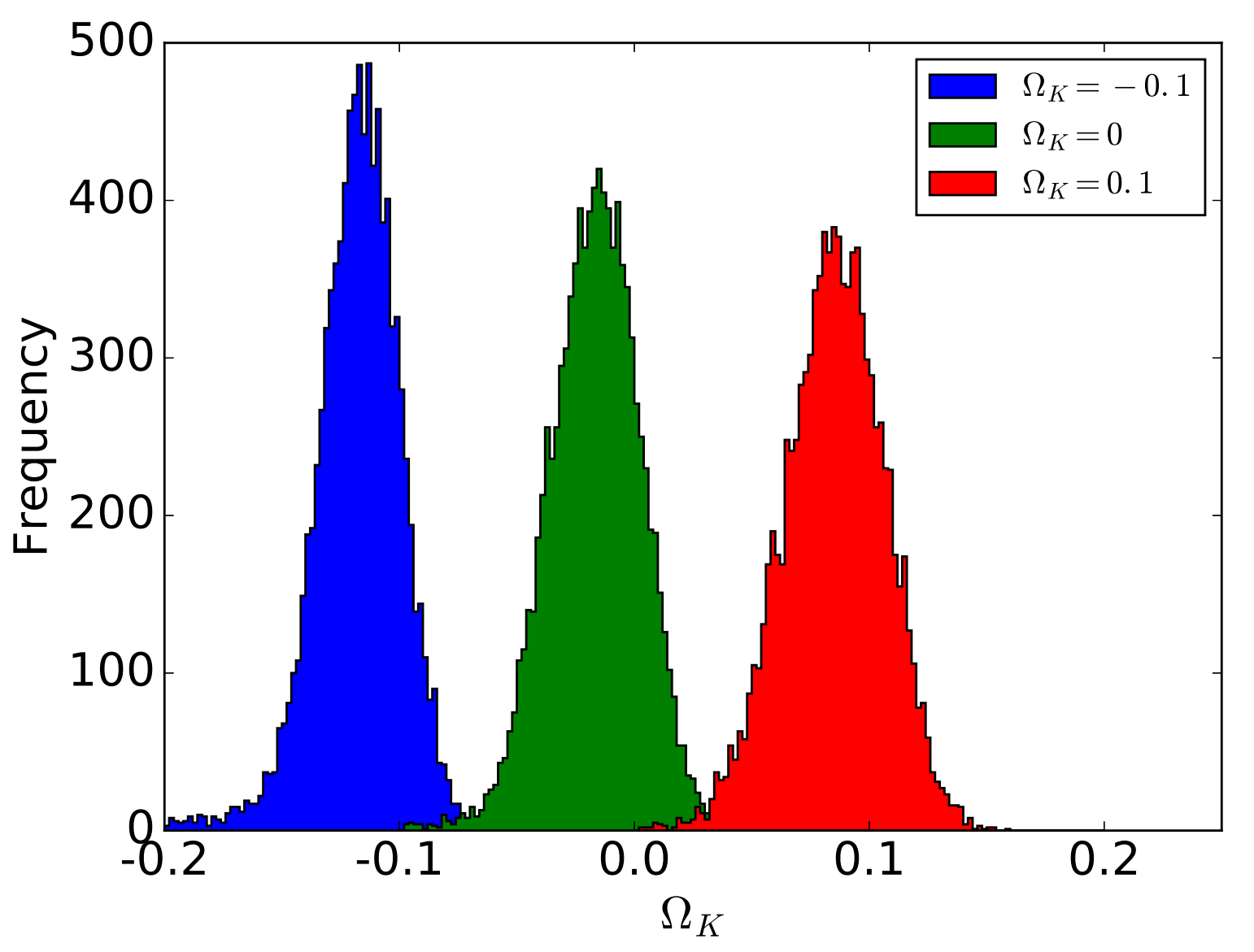

Fig. 8. - Same as Fig. [ but for C07 method. 
Table 1: The angular diameter distance $d_{A}(z)$ and their references.

\begin{tabular}{ccc}
\hline$z$ & $d_{A}(z)(\mathrm{Mpc})$ & Reference \\
\hline 0.44 & $1205 \pm 114$ & \\
0.6 & $1380 \pm 95$ & Blake et al. (2012) \\
0.73 & $1534 \pm 107$ & \\
\hline 0.35 & $1050 \pm 38$ & $\underline{\text { Xu et al. (2013) }}$ \\
\hline 0.57 & $1380 \pm 23$ & $\underline{\text { Samushia et al. (2014) }}$ \\
\hline 2.34 & $1662 \pm 96$ & Delubac et al. (2015) \\
\hline
\end{tabular}


Table 2: The data of Hubble parameter $H(z)$ and their references.

\begin{tabular}{ccc}
\hline$z$ & $H(z) \mathrm{km} / \mathrm{s} / \mathrm{kpc}$ & reference \\
\hline 0.09 & $69 \pm 12$ & Jimenez et al. (2003) \\
\hline 0.17 & $83 \pm 8$ & \\
0.27 & $77 \pm 14$ & \\
0.40 & $95 \pm 17$ & \\
0.90 & $117 \pm 23$ & Simon et al. (2005) \\
1.30 & $168 \pm 17$ & \\
1.43 & $177 \pm 18$ & \\
1.53 & $140 \pm 14$ & \\
1.75 & $202 \pm 40$ & \\
\hline 0.24 & $79.69 \pm 3.32$ & Gaztañaga et al. (2009) \\
0.43 & $86.45 \pm 3.27$ & \\
\hline 0.48 & $97 \pm 62$ & Stern et al. (2010) \\
0.88 & $90 \pm 40$ & \\
\hline 0.179 & $75 \pm 4$ & \\
0.199 & $75 \pm 5$ & Dlake et al. (2012) \\
0.352 & $83 \pm 14$ & \\
0.593 & $104 \pm 13$ & Moresco et al. (2012) \\
0.680 & $92 \pm 8$ & \\
0.781 & $105 \pm 12$ & \\
0.875 & $125 \pm 17$ & \\
1.037 & $154 \pm 20$ & \\
\hline 0.44 & $82.6 \pm 7.8$ & \\
0.60 & $87.9 \pm 6.1$ & \\
0.73 & $97.3 \pm 7.0$ & \\
\hline 0.35 & $84.4 \pm 7.0$ & \\
\hline 0.07 & $69 \pm 19.6$ & \\
0.12 & $68.6 \pm 26.2$ & \\
0.20 & $72.9 \pm 29.6$ & \\
0.28 & $88.8 \pm 36.6$ & \\
\hline 0.57 & $93.1 \pm 3.0$ & \\
\hline 2.34 & $222 \pm 7$ & \\
\hline & & \\
\end{tabular}


Table 3: The $\Omega_{K}$ derived from equation (5) using our method.

\begin{tabular}{lccccccc}
\hline$z$ & 0.35 & 0.44 & 0.57 & 0.60 & 0.73 & 2.34 & Average \\
\hline$\Omega_{K}$ & $1.24 \pm 2.78$ & $0.63 \pm 3.74$ & $0.29 \pm 0.75$ & $-0.28 \pm 1.59$ & $0.13 \pm 1.18$ & $-0.13 \pm 0.20$ & $-0.09 \pm 0.19$ \\
\hline
\end{tabular}

